\title{
Editorial
}

\section{Percutaneous myocardial laser revascularisation}

In the vast majority of patients with angina pectoris caused by underlying coronary artery disease, effective treatment is available. Most patients respond to antianginal medication, and for the remainder either percutaneous coronary revascularisation or coronary artery bypass grafting can be performed. There are, however, an increasing number of patients who have angina which is not controlled by medical treatment and have disease which is not suitable for conventional revascularisation techniques. Typically, such patients have atherosclerotic disease throughout their coronary arterial tree, with no "target" lesions for angioplasty and no "target" vessels for surgery. In recent years, transmyocardial laser revascularisation (TMLR) has been evaluated as a treatment option in this patient population. This technique, which is usually performed through a left anterolateral thoracotomy, uses laser energy to create transmural channels in the ischaemic myocardium. The initial experience with TMLR used a high energy carbon dioxide laser. As a result of developments in fibreoptic technology and by using a Holmium-YAG energy source, it is now possible to produce laser ablation from the endocardial surface of the left ventricle. This technique of percutaneous myocardial revascularisation has been investigated in randomised clinical trials.

\section{Transmyocardial laser revascularisation}

In the 1980s Mirhoseini reported the early experience with TMLR. ${ }^{12}$ The laser probe is placed on the surface of the ischaemic part of the left ventricle and activated. This creates a transmural channel into the left ventricular cavity. In an uncontrolled trial of TMLR in patients with refractory angina and coronary artery disease which was unsuitable for conventional revascularisation, three quarters had an improvement in symptoms of two angina classes. ${ }^{3}$ The findings from uncontrolled registry data, involving over 900 patients, showed that almost $50 \%$ experienced an improvement in angina of two classes. ${ }^{4}$ However, the in-hospital mortality for these studies was $9 \%$ and $9.7 \%$, respectively. In addition there was significant morbidity associated with the procedure, including infection (usually respiratory or wound), transient arrhythmia (typically atrial fibrillation), and left ventricular failure.

There have been two large randomised controlled trials of TMLR - one from the United States and one from the United Kingdom. The US trial randomised 198 patients to either continued medication or TMLR plus medication. ${ }^{5}$ There was a high crossover rate from medical treatment to TMLR and the 12 month data include only 64 patients from the TMLR group and 23 from the control group. An improvement of at least two angina classes was found at 12 months in $72 \%$ of the TMLR group and $13 \%$ of control patients. There was an operative mortality of $3 \%$ for TMLR with no significant difference in survival at 12 months between the two groups. The results of the UK trial were less favourable. ${ }^{6}$ In this study 188 patients with severe angina associated with coronary artery disease unsuitable for conventional revascularisation were randomised to receive either continued medication or TMLR plus medication. There were no crossovers and almost complete follow up data. At 12 months, the Canadian Cardiovascular Society (CCS) angina score decreased by at least two classes in $25 \%$ of the TMLR patients and $4 \%$ of the control group. There was a perioperative mortality of $5 \%$ for TMLR with no significant difference in survival at 12 months between the two groups. The morbidity associated with the TMLR procedure included wound/ respiratory infection (33\%), transient arrhythmia (usually atrial fibrillation) (15\%), and left ventricular failure (12\%). Exercise capacity measured using treadmill exercise times and 12 minute walk distance was not significantly different at 12 months between the two groups.

A recently published controlled trial using the carbon dioxide laser found symptomatic benefit at 12 months in patients with advanced ischaemic heart disease. ${ }^{7}$ Similar results were reported from a study using a Holmium-YAG laser. ${ }^{8}$ Both studies had limitations, including the high crossover rate from medical treatment to TMLR and incomplete follow up data.

TMLR, therefore, seems to produce symptomatic improvement in this patient population, although an increase in exercise capacity has not been consistently demonstrated. It carries a perioperative mortality of between $3-10 \%{ }^{3-6}$ as well as a significant procedural morbidity.

\section{Percutaneous myocardial laser revascularisation} Encouraged by the symptomatic benefits of TMLR, but conscious of the morbidity and mortality associated with the procedure, a less invasive technique has been developed. The initial experience with the catheter based approach, percutaneous myocardial laser revascularisation (PMR), was encouraging. ${ }^{9}$ The CardioGenesis system uses Holmium-YAG laser energy to create endomyocardial channels in the ischaemic part of the left ventricle. A 9 French aligning ("guiding") catheter is positioned within the left ventricular cavity. The laser delivery catheter is advanced through the aligning catheter and can be manipulated within the left ventricular cavity to reach the "target area". The laser delivery catheter carries an extendible laser optical fibre which is advanced to rest on the endocardium. The target areas for treatment are determined before the procedure from the results of the nuclear myocardial perfusion scan in conjunction with the angiographic findings. The procedure is carried out under local anaesthesia and requires the use of two radiographic projections (typically $40^{\circ}$ right anterior oblique and $50^{\circ}$ left anterior oblique with $10^{\circ}$ cranial angulation) to enable the precise "mapping" of the channels which have been created. A more sophisticated positioning technique (Biosense system) has been developed, which also uses 
Holmium-YAG laser energy. The CardioGenesis system uses two bursts of laser energy to create a channel $6 \mathrm{~mm}$ deep into the myocardium. Therefore, left ventricular wall thickness has to be at least $8 \mathrm{~mm}$ in the region to be treated.

The results of a multicentre randomised prospective trial of PMR (PACIFIC trial) have recently been reported. ${ }^{10}$ The study included 221 patients from several US sites and one UK site. The patients had angina refractory to medical treatment with disease not suitable for conventional revascularisation-111 patients were randomised to medication alone and 110 to PMR and usual medication. There was no crossover in the study, which involved detailed follow up of patients for one year. There was an improvement in angina at six and 12 months in the PMR group. The CCS angina score fell by a mean of 1.4 classes in the PMR group and 0.25 classes in the control group at six months $(p=0.001)$. The results at 12 months were similar. Exercise capacity also increased significantly in the PMR group. Treadmill exercise time increased by $30 \%$ in the PMR group and only $5 \%$ in the control group at six months $(p<0.001)$ - the baseline values were around 400 seconds in both groups. This improvement was maintained at one year. ${ }^{11}$ There were no in-hospital deaths in the patients undergoing PMR and the morbidity associated with the procedure was low. Of the 110 patients who underwent PMR, one developed cardiac tamponade requiring percutaneous drainage and one developed atrioventricular block which required permanent pacing. The costs of PMR are substantially less than TMLR, partly owing to the shorter in-hospital stay. Following PMR, patients are usually allowed home the following day, in contrast to TMLR when patients may be in hospital for up to 10 days. $^{6}$

In summary, therefore, TMLR seems to provide symptomatic improvement in this group of patients with refractory angina and no option of conventional revascularisation. There has not been a consistent increase in exercise capacity although this may, at least in part, be caused by the adverse effects of a thoracotomy in these patients with advanced cardiorespiratory disease.

The evaluation of myocardial perfusion before and after TMLR has also failed to demonstrate consistent improvement and the exact mechanism of action of TMLR remains uncertain. The evidence that TMLR increases angiogenesis, however, continues to accumulate. ${ }^{12}$ TMLR does carry a significant morbidity and this needs to be weighed against the potential benefits in each individual patient.
From the results to date, it is difficult to justify the widespread use of TMLR as a "stand alone" procedure in these patients. It may well have a role as an adjunctive procedure during coronary artery bypass surgery when complete revascularisation cannot be achieved using bypass grafts. ${ }^{13}$ In this context, since the patient is already undergoing major cardiothoracic surgery, the risks of TMLR are diluted.

The early results with PMR using a Holmium-YAG rather than a carbon dioxide laser seem encouraging. The six month and 12 month results from the PACIFIC trial ${ }^{811}$ have demonstrated symptomatic improvement and increased exercise capacity. The risk:benefit ratio for PMR appears to be much more favourable than that for TMLR. In future, it may be feasible to combine PMR with intramyocardial delivery of angiogenic peptides to induce further new blood vessel formation (neovascularisation).

SARAH C CLARKE PETER M SCHOFIELD

Regional Cardiac Unit,

Papworth Hospital,

Cambridge CB3 8RE, UK

1 Mirhoseini M, Fisher JC, Cayton MM. Myocardial revascularisation by laser: a clinical report. Lasers Surg Med 1983;3:241-5

2 Mirhoseini M, Shelgikar S, Cayton MM. New concepts in revascularisation of the myocardium. Ann Thorac Surg 1998:45;415-20.

3 Hovarth K, Cohn LH, Cooley DA, et al. Transmyocardial laser revascularisation: results of a multi-centre trial with transmyocardial laser revascularisation used as sole therapy for end stage coronary artery disease. 7 Thorac Cardiovasc Surg 1997;113:645-54.

4 Burns SM, Sharples LD, Tait S, et al. The transmyocardial laser revascularisation international registry report. Eur Heart $\mathcal{F}$ 1999;20:31-7.

5 March RJ. Transmyocardial laser revascularisation with the $\mathrm{CO}_{2}$ laser: one year results of a randomised controlled trial. Seminars in Thoracic and year results of a randomised control
Cardiovascular Surgery 1999;11:12-18.

6 Schofield P M, Sharples LD, Caine N, et al. Transmyocardial laser revascularisation in patients with refractory angina: a randomised controlled trial. larisation in patients with
Lancet 1999;353:519-24.

7 Frazier OH, March RJ, Horvath KA. Transmyocardial revascularisation with a carbon dioxide laser in patients with end-stage coronary artery disease. N Engl f Med 1999;341:1021-8.

8 Allen KB, Dowling RD, Fudge TL, et al. Comparison of transmyocardial revascularisation with medical therapy in patients with refractory angina. $N$ Engl F Med 1999;341:1029-36.

9 Oesterle SN, Reifart NJ, Meier B, et al. Initial results of laser-based percutaneous myocardial revascularisation for angina pectoris. Am f Cardiol 1998; 82:659-62.

10 Oesterle SN, Yeung A, Ali N, et al. The CardioGenesis percutaneous myocardial revascularisation (PMR) randomised trial: initial clinical results [abstract]. Am f Cardiol 1999;33(suppl A):380A

11 Oesterle SN, Ali N, Sanborn TA, et al. Percutaneous myocardial laser revascularisation (PMR): final results from the PACIFIC trial [abstract]. Circulation 1999;100:I-304.

12 Yamamoto N, Kohmoto T, Gu A, et al. Angiogenesis is enhanced in ischaemic canine myocardium by transmyocardial laser revascularisation. $\mathcal{f} \mathrm{Am}$ Coll Cardiol 1998;31:1426-33.

13 Clarke SC, Schofield PM. Myocardial laser revascularisation. Eur Heart $\mathcal{F}$ 1999;20:1213-14. 